\title{
Guidelines for Designing Mobility and Orientation Software for Blind Children
}

\author{
Jaime Sánchez and Miguel Elías \\ University of Chile \\ Blanco Encalada 2120, Santiago, Chile \\ \{jsanchez, melias\}adcc.uchile.cl
}

\begin{abstract}
We present a study about the use of current electronic travel aids to help blind people navigate through familiar and unfamiliar environments. We also discuss the main strengths and weaknesses of electronic travel aids and propose guidelines to design and use them adequately. We provide a proposal to develop and use traveling aids. As a result, this can be a first step towards defining major aspects to develop travel aids oriented to assist mobility and orientation of blind people.
\end{abstract}

Keywords: Mobility and Orientation, User-centered software development, Blind users, Electronic travel aids, Virtual environments.

\section{Introduction}

Software design for users with special needs entails strict requirements and user involvement. We cannot test software for people with visual disabilities by just blindfolding ourselves and other sighted users and try to mimic their interaction with the interfaces. A sighted user can not simulate the behavior of a blind user because their cognition and mental models are not alike [17]. Thus their feelings and tastes when interacting with digital devices are not really predicted. Therefore, when developing software interfaces for people with visual disabilities, additional efforts from designers and programmers are required. They have to understand the way blind users mental models work by continuously testing them throughout the development process.

Currently, in spite of diverse efforts [3], [7], [8], [9], [10] made to provide blind people with aids to support their navigation through indoor or outdoor environments, research on how and why to do this is scarce. Some existing aids are very sophisticated and use top of the line technology [9], [20], but not necessarily are targeting the blind user's needs and interests. Some of them are additional burden for the visually impaired. As a result, there is a clear shortage of usability studies on these issues and a lack of studies concerning the real impact of these aids on mobility and orientation performance of blind users.

This research presents traditional mobility aids and the skills involved when navigating. We introduce Electronic Travel Aids (ETAs) and discuss the main troubles with current designs. As a result of this analysis, we present guidelines for 
developing software for people with visual disabilities with special emphasis on supporting Mobility and Orientation (M\&O) skills by illustrating and classifying the main aspects involved and discussing the concretion of these guidelines in software design for blind people.

Electronic Travel Aids are assistant devices designed to support blind users during navigation through indoor and outdoor environments. They span from talking compasses to complex devices that make use of magnetometers and inertial sensors such as accelerometers and gyroscopes. ETAs comprise that employ sound beacons, ultrasonic pulse-echo sonar systems, infra-red detectors, among others [3], [7], [9], [10], [15], [20].

Many efforts have been made to complement or even replace the most employed and successful traveling aid so far: the white cane, to support blind people when traveling and avoiding obstacles by using the available technology (like GPS, RFID and WI-FI). ETAs usually have been developed by targeting on specific problems, but generally they provide an obstacle-avoiding system, location and orientation information, and optimal routes calculations during navigation [14].

The main drawback of existing ETAs is that current developments are not usercentered. This implies a series of usability and interaction issues, failing when providing solution to travel issues faced by blind people when traveling, and even creating new problems to them.

One of the main problems of current devices is the way information is presented to users. They do not present relevant information in a way users can actually be oriented and sometimes overwhelm users with a huge amount of information. For instance, some ETAs show a potential cognitive overload by presenting more than $7 \pm 2$ concepts on each interface, which is recommended for short-term memory processing issues and retention capacity [16]. This may be a consequence of a general understanding that, due to its difficultness and dangerousness for blind people to move around without perceiving well their surroundings, travel aids should provide a sufficient amount of information about the environment to create a mental representation of the location. This caused that many early developed ETAs pursued to present as much information about the environment as possible. This turned out to be an excessive, confusing and unnecessary amount of information which only confused blind users [12].

Leonard [6] made an experiment with blind people about interaction with information concluding that what is necessary and useful in a traveling aid is to present just small amounts of information, from which blind people can improve their navigational performance. These pieces of data about the environment should be presented in such a way that does not interfere with the information already being gathered by the blind individuals. This means that the amount of information presented should be short enough to create a mental map of the environment, without causing cognitive overload. Also, it should not occlude the information that blind people use as primary clues for mobility and orientation.

Literature shows that efforts in ETAs research aimed mainly at obstacle detection and avoidance, but as Loomis et al. [11] indicate, these devices have failed in contributing to the accomplishment of a more efficient navigation. The main reason is that providing a way of improving the navigation of blind people depends on information that is beyond the reach of these devices. Many studies fall short in 
determining what are real needs of blind people when navigating through familiar and unfamiliar environment. In some cases researchers have not been able to detect a need for a specific kind of information [20].

One of the most important issues of current ETAs is the lack of usability evaluations to validate the design of the interfaces being used by blind people. For instance, some devices utilize a set of headphones affecting the hearing perception of blind individuals and upon which they base most of their navigation. Other devices require that users utilize both hands to operate them, thus impeding blind individuals to use a cane or self-protection techniques.

Some devices are simply not designed for blind people and require so much learning and training (like reduced Braille and QWERTY keyboards), that they become an additional obstacle to blind users. Furthermore, there are some proposals of using big and heavy robots to guide blind people who ultimately present an additional barrier for them when taking the stairs or walking through narrow hallways. As Vogel [21] states, “...traditional navigation aids for the blind can be inconvenient to port, can be customized only with difficulty and manually, and usually cannot indicate the user's location...", p.2. In addition, formal evaluations of the impact of using one particular device for mobility and orientation of blind individuals are not frequent. This means that the real value of a particular ETA to improve the quality of life of a blind person is neither quantified nor determined.

Many ETAs research consist of highly customized devices that aim to solve a particular navigation problem. Usually they focus on the technology involved rather than in the real problems of blind people when traveling through an unfamiliar environment. One common agreement in this field is that there is no standard solution for the indoor navigation problem [7]. This leads to several unsolved problems, raising the cost of customized devices, thus making it more a barrier than a solution. Unfortunately, this widens the gap between blind people and technology. People with visual disabilities do not feel that the marginal mobility improvement provided by ETAs is worth the high price of these devices.

As a consequence, we propose to consider a more practical approach, centered on the needs and problems of blind individuals when navigating through unfamiliar environments and considering low-cost technology already tested successfully with people with visual disabilities, such as PDAs [18].

The literature proposes some elements to be considered when developing traveling aids for blind people. Loomis \& Golledge [10] state that the most significant needs of visually impaired people when navigating can be summarized in: accessing to information, accessibility to the environment, and independence when navigating.

Accessing to information with their remaining senses can be achieved by devices that have multimedia features, such as audio and haptic interfaces. These devices should focus on providing information that users can not obtain by their own means. Alternative displays such as Braille labels or tactile maps can also be used when presenting information.

Accessibility means not only that blind individuals can enter and move around inside a particular environment, but also implies they will be able to recognize landmarks, understand the layout of its components and navigate safely.

Independence when navigating implies that we should focus ETAs as $\mathrm{M} \& \mathrm{O}$ aids to help blind users to be more independent human beings. This can be considered from 
different points of view. First, there is no need for providing a traveling aid that can be an additional trouble for the blind individual. Therefore, it should make navigation easier, not demanding. This is the reason why we think that complex systems, such as robots or laptop-based devices, are not the right way to go, since their size and weight are a burden to users. Second, we feel that most devices should have a bounded life cycle. This means that the technology involved should support the acquisition and development of $\mathrm{M} \& \mathrm{O}$ abilities, not replace the human processes involved. The goal is to enhance an independent navigation of blind individuals through the usage of mobile. The role that an electronic aid can play in navigation should decrease as the individual develop more $\mathrm{M} \& \mathrm{O}$ skills and strategies. Third, a person with visual disabilities should not depend on other people (sighted or blind) to navigate a surrounding environment.

According to the Colorado Department of Education [4], blind students can benefit from understanding the physical environment, orienting to different school and community environments, traveling in school and in the community and finding opportunities for unrestricted, independent movement and play. This means that blind students should be able to learn, understand, and mentally represent the environment. They should be capable of comprehending the main elements in a particular type of indoor environment such as a school, a shopping mall or a public building, and their uses and functions. They should also be able to navigating them, travel to and from common and crowded places (like the cafeteria), and experiment new environments widening their $\mathrm{M} \& \mathrm{O}$ skills and knowledge. In a preliminary approach [8], people with visual disabilities envisioned $\mathrm{M} \& \mathrm{O}$ aids that augment their perception of the environment, announced points of interest located out of the reach of users and helped them to navigate through those points, and filtered objects according to a certain classification.

Cox \& Dykes [5] stated that some of the relevant skills to be developed by blind children inside the school environment are related to knowing landmarks, such as classrooms organization, common sectors (libraries, cafeteria), and knowing the location of entrances, exits and main offices. Hub et al. [7] mentioned that, according to some surveys carried out with blind individuals; these people have several problems with stairs, irregularities of the ground, and doors localization. This is stressed with their inability to realize the purpose of some rooms and buildings.

\section{Methodology}

We conducted several interviews in Santiago, Chile, with M\&O specialists, blind children and youngsters, to gather their opinions and feelings about the issues and difficulties that blind children face on a daily basis when navigating indoor environments. We asked them questions that allowed to understand how blind children travel to and from the school, how they move inside it, what problems they face when navigating in their schools and in other indoor environments, and what opinion they have about including blind children to traditional schools.

Scenario. Six interviews were conducted in Santiago, Chile, from March to October, 2006. The interviews took place in several locations, involving two types of schools: traditional (those designed for sighted children) and special (differentiated or 
segregated as they are commonly named). One interview was made at the Center for Computing and Communication for the Construction of Knowledge of the University of Chile.

Sample. The participants were very diverse, five blind youngsters (ages between 20 and 32 years old) and seven blind children (ages between 8 and 14 years old). Five children attended a special school and the other two attended traditional schools. Three M\&O specialists also participated; one of them is currently the teacher of M\&O courses at the Metropolitan University of Educational Sciences. The other two specialists are in charge of the $\mathrm{M} \& \mathrm{O}$ courses taught in a segregated school for the blind. Additional participants were two special education teachers, experts on vision disorders, three teachers and three educational psychologists from two traditional schools that integrate blind students.

Instruments. Diverse open interviews were applied for students, teachers and parents. All of them were unstructured interviews based on a previously established guideline with varying number of questions. The answers and comments made during interviews were recorded and saved in audio files. The interviewers also took written observational notes during the meetings.

Procedure. We conducted a first interview in March of 2006, at the Center for Computing and Communication for the Construction of Knowledge of the University of Chile. The participants were five blind adolescents, two special education teachers (experts on vision disorders), and three computer science engineers. The focus of that interview was on the problems that blind youngsters had when facing familiar and unfamiliar environments, and how they travel from one location to another, what kind of public transportation they currently use, what strategies they have when facing different problems, etc.

In June of 2006 the teacher of the M\&O courses at the Metropolitan University for Education Sciences was interviewed. She explained what the M\&O courses are, and how they teach them to in-service special teachers that will later work with blind people. During the same month two teachers from the School for the Blind "Santa Lucía", were also interviewed. They were in charge of the M\&O courses taught in that school. They provided information about the problems they face every day with blind children as their students, what landmarks they use as reference points, what strategies are used by children when they face problems, and what kind of information they think would be useful to deliver by an electronic travel aid. Later, another school, the private school "The Maisonnette", was contacted. They have integrated a blind girl into their school. We interviewed two of her teachers, along with the curriculum coordinator. The focus of the interviews was on problems faced by the blind girl when moving through the school, what pedagogical changes they had to do in their classes, and the impact of incorporating a blind child as student. We also had the opportunity of observing the blind child in one class and her navigation through the school's yard during breaks.

In July of 2006 we interviewed three educational psychologists of the "Lastarria" high school, a public institution that has integrated three blind children. After that we also met and interview one blind student. The focus of the interview was the same as in the other interviews previously described. In September of 2006 we interviewed 
five blind children (four of them with loss of vision) that attend the "Santa Lucía" School for the Blind. We asked them about the problems they face when navigating and what landmarks they use to orient themselves.

We finally made an in-depth interview on October of 2006 with the blind girl from "The Maisonnette" School, in her house. We talked with her mother, who mentioned the problems faced when her daughter navigates indoor environments and the efforts made to integrate her in a school for sighted children. Later, we talked to her, a 9 years old child who told us about how she moves inside her school, what problems she faces (such as route planning and getting lost), and how a guiding digital device should look like. All the information we gathered from these interviews were analyzed and summarized in the following section.

M\&O specialists were asked about the type of information they think should be useful to provide to blind children during their navigation through the school environment. They mentioned that the most important information was the environment corners, middle points, and the location of every day visited places, such as bathrooms and exits. They mentioned that is also important to provide to blind people contextualized information of the surroundings (cardinal location of sidewalks, public transportation). The main difficulties of blind people when navigating through indoor environments pointed out by specialists were the detection of obstacles beyond the scope of their primary mobility aids (canes or even their own body). Lintels, beams, chandeliers and arches are examples of these obstacles.

When we asked blind youngsters about their problems when navigating indoor environments, they agreed that they frequently collide with obstacles not detected by their primary mobility aids. Some examples of these obstacles are stairs and entrances with low-height steps (so the cane passes right over them). When inquired about problems in their navigation within the school, blind children said that they were mainly concerned about colliding with a wall or other people, and the chance of changing the landmarks currently used to other unknown places, revealing that sometimes they use temporal information for navigation.

Blind people want to know where they are and what objects are close to them, but they do not want other people to tell them about everything. When augmenting their reality by means of artificially enhancing their perception we should opt to provide them information which cannot perceived autonomously. Once the blind person knows where he or she is, the next step is helping him or her to plan a route, providing the necessary information so he or she can make an informed decision on what legs to follow. This is obtained not only by providing information of landmarks and paths in advance, but also by providing additional information such as the cost of each path (in terms of their lengths and hazards).

According to Baldwin [2], teaching routes should have a concrete purpose to make learners able to travel to places of their interest. This is complemented with a goalorientated strategy. After the student is able to travel a certain route it is suggested to revert it and increase the number of landmarks. It is also recommended to start with straight routes on which rotations can be included incrementally.

We should not make the decision for them when presenting ways of getting to a place. Sometimes people (blind or sighted) may choose a path that does not appear to be "optimal", but that may have hidden benefits that influence their decisions. They can actually figure out shortcuts and navigate them, but sometimes their decisions rely 
on factors beyond saving time. They may prefer to use a less cognitive demanding route, less crowded, with more easily detectable landmarks, and less environmental noise (distracting sounds, absence of reference cues). At times they even do not care about which route to take, even though the route chosen may be not the best route. As a blind Chilean student stated, she knows that the hallways of her school form a loop, so it does not matter which way to go, since she will always arrive safely. Blind students should develop the ability to choose between different navigational alternatives and make informed decisions.

Blind children have a restricted perception capacity as a consequence of their lack of interaction with the environment, provoked by their fear to explore and parents and teachers overprotection. They also have a weak body image and difficulties to understand spatial concepts [1]. These abilities should be developed early, altogether with motor coordination and pre-cane skills.

The lessons learned after our interviews suggest that computer systems should be designed to reduce the anxiety and stress that arise in blind navigators when dealing with unknown environments. A game-oriented approach can contribute to this objective, especially when having children as end-users. Blind children should be exposed to situations where they have to solve problems perceiving information and thinking individually at their own pace, and as a group working collaboratively in teams. This is why we suggest avoiding the development of a software solution that solves every single navigation problem; rather it should be an aid that allows them to solve problems by their own, encouraging the development and use of M\&O skills and abilities.

Finally, we should also consider the children's learning curve. We should start by using familiar elements with simple lessons before starting more complex and challenging schemes. We should keep in mind that the earlier a blind child starts an $\mathrm{M} \& \mathrm{O}$ program, the sooner he or she would become independent and able to navigate independently. In cases when the child does not have the appropriate neuromuscular development to use of a mobility aid such as the cane, an ETA can be an appropriate aid.

Landmarks. When teaching routes to blind children (and blind individuals in general), the use of landmarks is essential. They are references within an environment that allow blind individuals locating elements in their precise position (doors and stairs) and approximate position (smell or sound). Information provided by a landmark allows finding a way and through the memorization of references, to easily rebuild a route when a landmark appears in the path. A customization feature should be provided when using landmarks. Each individual utilize different sets and combinations of textures, smells and sounds to orient themselves. What is useful for one person could not be useful to others, even not recognizable by most blind users.

The interviews with blind children evidenced different positions concerning landmarks for mobility and orientation purposes. While some of them stated that they use landmarks (such as water fountains, light variations and smells) on a daily basis, others said that they did not use landmarks while navigating (at least consciously). We believe that the latter group is not aware of the real importance of understanding and utilizing landmarks for navigational purposes. The literature stated that landmark information combined with an audio interface can enhance the mental mapping of the 
blind user [12]. Therefore, we should focus our efforts in developing travel aids that support training and utilization of landmarks, since this would be most beneficial for blind children navigation.

\section{Guidelines for Developing Software for Context Navigation}

The development of software for people with visual disabilities can be a great challenge for designers, since it implies a multidisciplinary approach. Many issues mentioned can be overcome by following a series of guidelines designed from an integration of recommendations suggested by researchers and our own formal observations previously described, that should be considered when developing software for blind people, especially for navigation of blind children. We also present some specific guidelines for developing mobility and orientation software to train individuals in their navigation of familiar and unfamiliar virtual environments and during online guidance and support when navigating real environments.

Model. In the context of M\&O software we would expect to be scalable, flexible, and adaptable. One approach is to use validated models in similar contexts in the past and adjust them to current needs. Then, we want to ensure that children will actually use that aid. This can be achieved by designing software appealing and encouraging, thus motivating its use. Games and playful activities could fit well this need. Besides how attractive or entertaining is the software it has to be usable. Therefore, usability evaluations should be mandatory. Sánchez \& Baloian [19] mentioned that when developing software for blind users, one of the most important guideline is that the software should be based on a model, game-oriented and consider formative ans summative usability evaluations.

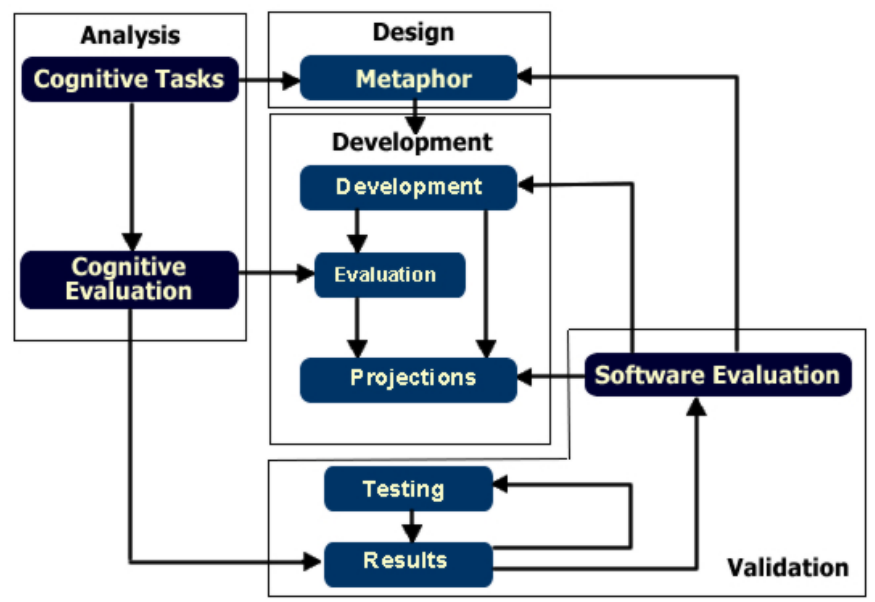

Fig. 1. Model of software for blind children (from Sánchez, J. \& Baloian [19]) 
A suitable model and methodology that can be adapted to $\mathrm{M} \& \mathrm{O}$ development is proposed by [19], used for designing and developing audio-based games for blind children. In this model (see Figure 1), a combination of incremental and evolutionary development is proposed considering the stages of analysis, design, development, and validation, by making particular considerations when developing educational software for blind children. We think that this model is pertinent for developing M\&O software for blind users, since it considers a prototyping approach that allows lowering the associated risk of failing to map the user's mental model, and can be adjusted to potential unrevealed requirements at a low cost.

The model proposed should also be adapted and instantiated to reflect new interactions and features arising from the use of software in mobile devices for $\mathrm{M} \& \mathrm{O}$ purposes. When passing from a desktop to a mobile application, the main adjustment is related to acknowledge the role played by the environment: it is dynamic and evolutionary, and triggers the user's actions.

Game orientation. By considering children as end-users the software should be game-oriented to let them to learn in a playfully way. By combining learning and entertainment (edutainment) we can provide a dynamic and appealing experience, thus enhancing a more effective learning. Gaming is an interesting way of achieving a higher commitment of learners with the learning process due to the emotional involvement of the player during the game. Games are a good and attractive way of practicing higher order skills such as problem solving. The model suggested is gameoriented emphasizing cognitive tasks embedded in the software through a game metaphor interface.

Evaluation. The proposed model considers the use of formative and summative endusers usability evaluations. This is fundamental to gather data about the users' acceptance and how well the software meets their mental models. By considering methods such as heuristic evaluations and cognitive walkthroughs, hidden requirements are highlighted to improve the user's interaction. Virtanen \& Koskinen [20] stated that the evaluation of a device should not be made in terms of the information provided; rather the focus should center on the real benefits produced. Children should practice with the applications in real environments and contexts, thus assuring safe and natural activities.

Interfaces. User interfaces should be intuitive and suitable. The best possible interaction models to select type, quantity and ways of presenting information should be used [21]. Users should have modes of customizing the presentation of information according to their needs and interests. When having people with visual disabilities as users, particularly those who have loss of vision, interfaces should be designed in ways to fully exploit the remaining visual channel to perceive information. When the interface includes text (in menus and labels), sans serif fonts should be considered, since the resolution of computer screens is lower than paper. This makes screen reading easier and less exhausting. A clear interlining spacing and highly contrasting colors (such as blue/yellow or black/white combinations) should be also considered.

Iconic photographic images should be preferred. It is most likely that people with visual disabilities are not able to appreciate small details of an image (such as facial features of a character). Instead, simple and clear icons should be used. Icons must 
have good contrast with the background color and should be related with audio clues to let users comprehend their functionalities. Audio cues must be included so users can construct a mental model of the virtual environment, and thus improving the interaction.

Interaction. Different ways of navigating the virtual environment should be provided. This encourages users to explore several traveling strategies and the construction of knowledge about routes, locations and timings. Ultimately, this contributes to the construction of the mental representation of the virtual environment. Furthermore, exploring different navigational strategies and approaches in a controlled and safe virtual environment can be the first step to encourage blind individuals to do the same in real environments.

Users should be make decisions and take actions. They should be allowed to interact freely and directly with every element in the virtual environment. Even tough sometimes some type of guidance is necessary; users should be free to decide whether or not to accept the suggestions made. Each user's action should have an immediate and clear feedback, so he or she can realize permanently what has been done and what are the consequences of their actions, thus supporting cognitive interaction.

Audio cues. Audio is an essential channel to convey information to blind users. Good quality and easily identified audio should be used. Non spoken sounds are easier to listen than verbal audio cues [12], they do not distract users from navigation and can be easily ignored. Iconic sounds (sometimes called earcons) are appropriate to convey information, whether it is general, specific or quantitative. Earcons are also nondisruptive to users [21]. Although spoken sounds may be appealing and useful they can not be modified after recording. However, the use of earcons does not exclude the use of verbalized forms of audio. If spoken sounds are used to provide more complete description of landmarks, for instance, they can be more useful if we use cardinal coordinates in our descriptions, providing lengths by using step counting and $\mathrm{M} \& \mathrm{O}$ concepts (turns, quarter-turns).

Artificial sounds should not occlude natural sounds coming from the environment intake. One way to accomplish this is considering the "cocktail party effect" when providing audio cues in the presence of environmental sounds. This means that we can exploit the human ability of listening to several sound sources at the same time, without getting confused. This also implies users can pick one sound in particular, as humans do when several people are talking and find a conversation nearby to be of their interest.

Sound pinned to landmarks should be representative of the point of reference in such away that users can identify them through sound. For instance, if a water fountain is used as a landmark, a running water sound effect could be a good reference.

Monophonic and stereo sounds as primary navigational senses should be avoided since using them may block environmental sounds.

Content \& information. Information has to be available anytime time and users should be able to listen important messages whenever they need to do so. Sometimes the interaction can be asynchronous. If a user listen environmental audio cues such as messages coming from a speaker in the subway station, or if he or she talks to a 
friend, the information provided by the navigational aid can be missed. Outdoor designs should consider the asynchronous features of interaction and provide ways of repeating important messages in any moment.

Instructions should be clear and simple [21], since users have to remind them during interaction with the navigational software. This pursues to present information processed by users without distracting them from navigation.

The information provided by a navigational device should to be contextualized information. Not every piece of information available is useful for navigational purposes. Only a small subset of information should be presented to users according to their needs during navigation. ETAs should provide the orientation and position of users, their targets and landmarks to avoid cognitive overhead.

Every user is different to the other and sometimes a piece of information that is essential and relevant for one user can be completely incidental for another. Therefore, some degree of customization has to be considered. ETAs should be able to adapt to users, selecting adequately the hints provided to fit the needs and experience of the current user. Customization should be made by users through adapting the related software and provided as intelligent agents that learn and plan present and future users' needs.

Representation of the environment. Some elements should be repeated iteratively throughout different environments. For instance, most indoor environments are fairly rectangular or squared-shaped. Many of them have four walls, a floor and a ceiling, and at least one entrance/exit. One example of this type of environments is a classroom. Almost every classroom has a chalkboard, chairs, tables, and so on, therefore when students understand the layout and composition of one classroom, their knowledge can be generalized to the rest of classrooms. The same concept can be extended to other environments such as a mall, the zoo and the library. This idea can be complemented with the work done by Kàpic [8] who stated that to represent an environment and routes we should utilize a rectangular approach. Routes should be composed of straight segments that intersect each other in a 90-degrees angle and their lengths should be of not longer than ten meters (approximate 33 feet). If the right angle approach is not enough for a particular environment, then we can use clock navigation for directions.

The location and orientation of users should be known anytime anywhere. A way of detecting a missed path should be also provided.

Devices \& Infrastructure. Devices utilized should be small enough to be carried comfortably (in a palm hand or in the pocket). This means that the devices used should meet three main requirements. First, the device should not interfere with the primary mobility navigation aid used by a blind user, such as canes and guide dogs. Second, as many blind individuals declare, any M\&O aid should not highlight the blind user as a person with disabilities. This is one of the reasons why many blind children do not like using the white cane, since although this mobility aid has many advantages it socially shows them as blind users. The interfaces and size of the devices should also be suitable and comfortable enough for blind users.

Devices utilized in $\mathrm{M} \& \mathrm{O}$ contexts should have low battery consumption, so they can provide uninterrupted services for reasonable timeframes. Software designed for these devices should provide ways to alert users when battery is low loading. Many of 
these devices manage databases and I/O features (microphones, speakers or earphones). This implies that there is a minimal processing power required for these devices. According to Kàpic [8], devices should have a monitoring system embedded that provides information about the remaining battery and processing capabilities.

Users should be able to receive and utilize location-dependent services not only from static repositories, but also from other users, designing the infrastructure in a scalable way to preserve users' privacy. The cost of the solution (considering devices and infrastructure) should be low and imply few changes in the current environment to make it replicable. One way of achieving this is using devices meant for other purposes, instead of building new ones from scratch. One option is the use of PDAs, which are increasingly being used in schools for educational purposes, thus lowering entrance barriers. PDAs comprise the required battery and processing capabilities, multimedia features, portability, and their cost is reasonable (compared to laptops). Cell phones also meet these requirements with the additional advantage of being worldwide massively distributed.

\section{Discussion}

Many efforts have been made to complement or even replace the white cane as an aid tool for blind people. ETAs developers should not focus on this "replacement" intent since it is very unlikely to redesign such a device that is cheap, portable and useful as the white cane. We should make efforts to complement this navigation aid rather than to replace it trying to change the primary navigation aids. This implies a device that can detect and inform users about obstacles and hazards beyond the reach of primary mobility aid (cane, guide dogs), and provide information about the environment to help blind people to develop a more accurate mental model of the surroundings.

Sometimes the problem is not the use of an aid but its misuse. Some specialists said that some blind individuals walk very fast and do not utilize the cane properly (they describe only a tight sweep, for instance), due to an excess of confidence or just because of trying to keep the pace of sighted people, missing some obstacles and thumping on them.

There is no such a product that can solve every single problem of every blind individual. This is not a pessimistic but a rather realistic affirmation. ETAs developers should not expect to solve all M\&O problems for every blind person. Instead, we believe that research studies should encourage and promote the development and utilization of $\mathrm{M} \& \mathrm{O}$ skills in blind people to achieve an independent, safe and efficient navigation, promoting problem-solving features of navigational aids.

It is essential to develop a deeper understanding about blind users' interest, needs and way of knowing. As Psathas [13] stated, people navigate mechanically so many behaviors and tasks are made unconsciously, being unable to verbalize current practices and issues involved during navigation.

The cost of ETAs should not be a barrier for a blind individual. Developers should consider low-cost technology fully tested with people with visual disabilities, such as PDAs, devices that can be used for many purposes (like educational software, collaborative work) besides to M\&O. Schools everywhere are starting to insert this kind of technologies into curriculum. Considering also the high social penetration of 
cell phones, it is most likely that in the short-term, most students will have a mobile device with the required capabilities to use them as ETAs. Therefore, the idea is to reuse existing devices and infrastructure for $\mathrm{M} \& \mathrm{O}$ purposes, ending up with a system that is cheap, available to blind people, that provides the information needed for navigation, allows them to understand the environment without the aid of a sighted person, has low impact on the existing infrastructure, and thus making feasible to implement it everywhere.

There is a clear need for guidelines when developing navigational software for blind users, especially for children. This is why we provide guidelines for developers that should be seen as the starting point to draw from their own experiences. For instance, we suggest that highly contrasting colors should be used, but if you use only three or four colors, children with residual vision will get bored of the interface. This is why we suggest trying new combinations of colors, images and media, starting from experience. But any design experience should be evaluated formally with real end-users, during a significant timeframe, using usability methods, and considering that it is very unlikely to come up with an interface that works for every user and suits every single need. This should be considered as a challenge to design interfaces that meet the interaction model of most blind users.

Interfaces for long-term interaction should consider that certain types of blindness are degenerative so designers should incorporate some evolutionary features to adjust itself to the loss of vision.

ETAs should not be an additional burden to blind people when navigating. This implies that blind users should be able to navigate autonomously, without the assistance of a sighted individual. The devices are portable and easy-to-use when walking through narrow hallways or taking the stairs. In addition, we should aim at letting users to diminish their dependency on the involved technologies as they develop, use and improve M\&O skills for navigation.

Emerson Foulke once said that we know more about what is involved in getting a man to the moon than about what is involved in getting a blind person to cross the street. As analyzed and discussed in this report, ETAs are far from being a final solution for navigational problems of blind people, but they are a very good starting point to help blind children to develop, use and rehearse mobility and orientation skills for a better social inclusion.

Acknowledgements. This report was funded by the Chilean National Fund of Science and Technology, Fondecyt, Project 1060797.

\section{References}

1. Arnaiz, P., Martínez, R.: Educación Infantil y Deficiencia Visual. Editorial CCS. pp. 66-97 (1998)

2. Baldwin, D.: Teaching Orientation and Mobility to Blind Children (e-Book), Chapter 3: Navigating without vision. Retrieved (October 2, 2006), from www.wayfinding.net/ play.htm 
3. Cheung, S., de Ridder, S., Fishman, K., Francle, L., Patterson, J.: A Personal Indoor Navigation System (PINS) for people who are blind. Retrieved (November 28, 2006), from vision.psych.umn.edu/ gellab/5051/prev_projects/pgs1.pdf

4. Colorado Department of Education. Orientation and Mobility as a Related Service. Retrieved (November 28, 2006), from: http://www.cde.state.co.us/cdesped/download/ pdf/OM_Related_Service.pdf

5. Cox, P., Dykes, M.: Effective Classroom Adaptations for Students with Visual Impairments. Teaching Exceptional Children 33(6), 68-74 (2001)

6. Heyes, T.: Electronic Travel Aids - Why Bother? Retrieved (August 21, 2006), from: web.aanet.com.au/tonyheyes/pa/quest.html

7. Hub, A., Diepstraten, J., Ertl, T.: Design and Development of an Indoor Navigation and Object Identification System for the Blind. In: Proceedings of the ACM ASSETS 2004, Atlanta, Georgia, USA, October 18-20, pp. 147-152. ACM Press, New York (2004)

8. Kàpic, T.: Indoor Navigation for Visually Impaired (Retrieved July 18, 2006) (2003) from www.mics.ch/SumIntU03/TKapic.pdf

9. Kulyukin, V., Gharpure, C., Nicholson, J., Pavithran, S.: RFID in robot-assisted indoor navigation for the visually impaired. In: Proceedings of IEEE/RSJ IROS 2004 Conference, September - October 2004, vol. 2, pp. 1979-1984. Sendai Kyodo Printing, Sendai, Japan (2004)

10. Loomis, J., Golledge, R.: GPS-Based Navigation Systems for the Visually Impaired. In: Barfield, W., Caudell, T. (eds.) Fundamentals of Wearable Computer and Augmented Reality, pp. 429-446. Lawrence Erlbaum Associates, Mahwah, NJ (2001)

11. Loomis, J., Klatzky, R., Golledge, R.: Navigating without vision: Basic and Applied Research. Optometry and Vision Science 78(5), 282-289 (2001)

12. Millar, D.: Spatial Audio Interface: Providing Landmark Information to Help the Blind Navigate Outdoors (Retrieved April 4, 2006) (2002), from www.cs.unc.edu/ vogel/IP/IP/ info/dorianm_fall2002.pdf

13. Psathas, G.: Mobility, Orientation and Navigation: Conceptual and Theoretical Considerations. New Outlook for the Blind 9, 385-391 (1976)

14. Ran, L., Helal, A., Moore, S.: Drishti: An Integrated Indoor/Outdoor Blind Navigation System and Service. In: Proceedings of the 2nd IEEE Pervasive Computing Conference, Orlando, Florida, pp. 23-30. IEEE Computer Society Press, Los Alamitos (2004)

15. Ross, D., Lightman, A., Henderson, V.: Cyber Crumbs: An Indoor Orientation and Wayfinding Infrastructure. In: 28th Annual RESNA Conference Proceedings (Retrieved July 7, 2006) from, www.cc.gatech.edu/ vlh/pubs/resna05.pdf

16. Sánchez, J.: Visible Learning, Invisible Technology. Dolmen Ediciones, Santiago (2001)

17. Sánchez, J., Sáenz, M.: 3D sound interactive environments for blind children problem solving skills. Behaviour \& Information Technology 25(4), 367-378 (2006)

18. Sánchez, J., Aguayo, F.: Mobile Messenger for the Blind. In: Stephanidis, C., Pieper, M. (eds.) EP 2007. LNCS, vol. 4397, pp. 369-385. Springer, Heidelberg (2007)

19. Sánchez, J., Baloian, N.: Modeling 3D interactive environments for learners with visual disabilities. In: Miesenberger, K., Klaus, J., Zagler, W., Karshmer, A.I. (eds.) ICCHP 2006. LNCS, vol. 4061, pp. 1326-1333. Springer, Heidelberg (2006)

20. Virtanen, A., Koskinen, S.: NOPPA: Navigation and Guidance System for the Blind. (Retrieved June 30, 2006), from virtual.vtt.fi/noppa/noppa\%20eng_long.pdf

21. Vogel, S.: A PDA-Based Navigation System for the Blind. M.S. Integrative Paper (Retrieved April 6, 2006) (2003), from http://www.cs.unc.edu/ vogel/IP/IP/IP_versions/ IPfinal_SuzanneVogel_Spring2003.pdf 